\title{
Sequential catalytic carbonylation reactions for sustainable synthesis of biologically relevant entities
}

\author{
Liliana Damas, Fábio M.S. Rodrigues, Andreia C.S. Gonzalez, Rui M.B. Carrilho*, \\ Marta Pineiro, Mariette M. Pereira ${ }^{1,{ }^{* *}}$ \\ University of Coimbra, Coimbra Chemistry Centre, Department of Chemistry, Rua Larga, 3004-535, Coimbra, Portugal
}

\section{A R T I C L E I N F O}

\section{Article history:}

Received 1 June 2020

Accepted 23 June 2020

Available online 15 July 2020

\section{Keywords:}

Sequential reactions

Aminocarbonylation

Hydroformylation

Microwave synthesis

$\mathrm{N}$-heterocycles

Chalcones

\begin{abstract}
A B S T R A C T
The sustainable synthesis of highly functionalised formylcarboxamide compounds with biological relevance is reported through a sequential aminocarbonylation/hydroformylation approach. The optimisation of palladium-catalysed aminocarbonylation of iodoaromatic substrates, using allylamine as nucleophile was first performed, with molybdenum hexacarbonyl as alternative CO source versus gaseous carbon monoxide. The combination of microwave irradiation with molybdenum hexacarbonyl allowed to selectively prepare a set of $N$-heterocyclic-based allylcarboxamides. Subsequent rhodiumcatalysed hydroformylation of the allylcarboxamide intermediates led to the preparation of new pyridine, pyrazoline and chalcone derivatives containing both carboxamide and formyl moieties.
\end{abstract}

(c) 2020 Elsevier B.V. All rights reserved.

\section{Introduction}

Carbonyl compounds, such as carboxamides and aldehydes, are among the most relevant synthons for preparation of highly functionalised molecules with potential applications in medicinal chemistry [1-6]. Particularly, those containing $N$-heterocycles [7-14] and chalcone [15-17] cores are frequently present in important pharmacological entities, such as antibiotics, antibacterial, anti-hypertensive, anti-inflammatory and anticancer drugs (Fig. 1).

Therefore, there has been an increasing interest in the search for efficient and sustainable chemical processes for preparing such molecules. Among them, transition metal catalysed hydroformylation [18-25] and aminocarbonylation [26-34] reactions are two paradigmatic examples of efficient one-pot and versatile synthetic approaches to get access to carbonyl compounds, such as aldehydes and carboxylic acid derivatives. Regarding these topics, it should be highlighted the relevant contribution of Kollár, with more than 300 published papers [35].

\footnotetext{
* Corresponding author.

** Corresponding author.

E-mail addresses: rui.carrilho@uc.pt (R.M.B. Carrilho), mmpereira@qui.uc.pt (M.M. Pereira).

${ }^{1}$ Dedicated to Professor Laszló Kollár, on the occasion of his $65^{\text {th }}$ birthday.
}

Furthermore, the development of sequential carbonylation reactions have led to significant reduction in costs, purification steps and waste formation. Such sequential reactions present higher atom economy, allowing to improve the general processes efficiency and sustainability [36-44]. It should be also noted that the global demands for implementation of more sustainable synthetic processes have boosted the search for alternative $\mathrm{CO}$ sources [45-49], including metal-carbonyl compounds [50-53] and the development of energetically favourable processes, such as microwave-assisted carbonylation reactions [54-58].

Along the last years, we have been focused on the development of efficient transition-metal catalysed carbonylation-based sequential processes applied to biologically relevant substrates for the preparation value-added products with biological activity and/or industrial interest, including hydroformylation/arylation [59], hydroformylation/isomerisation [60], hydroformylation/ reductive amination [61, 62], hydroformylation/Strecker [62], hydroformylation/hydrogenation [63] and aminocarbonylation/ cyclisation [64].

As part of our continuing research in this field, herein we report an original sequential process for preparation of biologically relevant formylcarboxamide compounds comprising $\mathrm{N}$-heterocycle and chalcone scaffolds, through microwave-assisted palladiumcatalysed aminocarbonylation of iodoaromatic substrates with allylamine, followed by rhodium-catalysed hydroformylation of the 


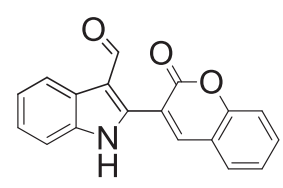

D. Sunil et al. ${ }^{[11]}$

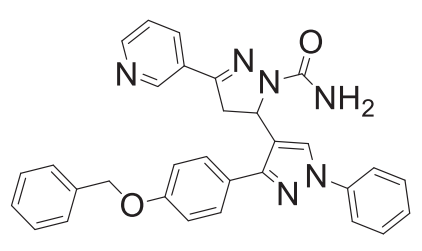

O. Alam et al. ${ }^{[12]}$

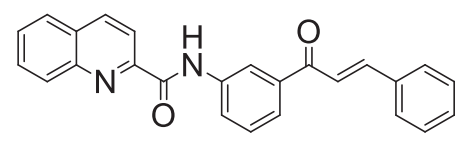

P. Vadivel et al. ${ }^{[15]}$

Fig. 1. Examples of $N$-heterocyclic and chalcone-based carbonyl compounds with pharmacological activity.

allylcarboxamide intermediates, to obtain carbonylated products containing both formyl and carboxamide motifs (Scheme 1).

\section{Results and discussion}

The palladium-catalysed aminocarbonylation step was first optimised, using iodobenzene (1) as a model substrate, allylamine as nucleophile, palladium (II) acetate as catalytic precursor, DBU as base and dioxane as solvent. The reactions were carried out in the presence of carbon monoxide or molybdenum hexacarbonyl as alternative $\mathrm{CO}$ carbonyl source, at $125^{\circ} \mathrm{C}$, using either conventional heating or microwave irradiation. Conversion and selectivity were determined by GC-MS analysis of the crude mixtures and the results are presented in Table 1.

Using a $\mathrm{CO}$ pressure of 3 bar and conventional heating, a conversion of $79 \%$ was obtained in $60 \mathrm{~min}\left(\mathrm{TOF}=32 \mathrm{~h}^{-1}\right)$, with $69 \%$ selectivity for the carboxamide product (2) (Table 1, entry 1 ). Under microwave irradiation ( $150 \mathrm{~W}$ ), a similar conversion was obtained in just 5 min, leading to a substantial increase in TOF $\left(365 \mathrm{~h}^{-1}\right)$, although a slightly slower selectivity (62\%) was observed (Table 1 , entry 2). Alternatively, the use of $\mathrm{Mo}(\mathrm{CO})_{6}$ complex as alternative carbonyl source, under conventional heating, provided full conversion in $60 \mathrm{~min}\left(\mathrm{TOF}=40 \mathrm{~h}^{-1}\right)$, with $82 \%$ selectivity for the carboxamide (2) (Table 1, entry 3 ). In addition, under microwave irradiation, the reaction proceeded with full conversion in $5 \mathrm{~min}$ (TOF $=475 \mathrm{~h}^{-1}$ ) and with $76 \%$ selectivity for the carboxamide product (2) (Table 1, entry 4). In sum, microwave irradiation allowed to significantly reduce the required reaction time from $60 \mathrm{~min}$ to $5 \mathrm{~min}$ with enhancement of the activity (TOF $=40$ and $475 \mathrm{~h}^{-1}$ respectively), maintaining the reaction selectivity.

With optimised conditions, microwave-assisted Pd-catalysed aminocarbonylation reactions, using $\mathrm{Mo}(\mathrm{CO})_{6}$ as carbonyl source, were applied to different iodoaromatic substrates with biologically relevant scaffolds, including 1-bromo-4-iodo benzene (3), 4iodoanisole (4), iodopyridine (5), 7-iodoindole (6), 5-iodoindole (7), iodopyrazoline (8) and iodochalcone (9) [68]. Conversion and yields were determined by GC-MS analysis of the crude mixtures and the results are presented in Table 2.

The microwave-assisted aminocarbonylation reactions proceeded with ca. 99\% conversion in 5 min and allylcarboxamide derivatives were obtained as major products in all cases, in yields ranging from $65 \%$ (for product 11 ) to $87 \%$ (for product 13 ). In addition, the aminocarbonylation of substrates $\mathbf{3}$ and $\mathbf{5}$ led to predominant formation mono-carbonylated products $\mathbf{1 0}$ and $\mathbf{1 2}$, respectively (Table 2, entries 2 and 4), which indicate that oxidative addition to the palladium complex occurs mainly through the most reactive $\mathrm{C}-\mathrm{I}$ bond, while $\mathrm{C}-\mathrm{Br}$ and $\mathrm{C}-\mathrm{Cl}$ bonds remain unchanged under these conditions. Moreover, in the case of iodopyrazoline (8), the reaction required $1 \mathrm{~h}$, under microwave irradiation, to obtain the corresponding allylcarboxamide product (15), obtained in $70 \%$ yield. In all cases, GC-MS indicated the formation of ketocarboxamides as minor products (up to $\mathrm{ca} .10 \%$ ), corroborated by the ${ }^{13} \mathrm{C}$ NMR spectra which presented typical signals around $190 \mathrm{ppm}$. After work up and purification by column chromatography in silica gel, using EtOAc/ $n$-hexane or EtOAc $/ \mathrm{CH}_{2} \mathrm{Cl}_{2}$ mixtures as eluents, the carboxamide products $\mathbf{2}$ and 10-16 have been isolated and fully characterised. In sum, microwave-assisted palladium-catalysed aminocarbonylation of iodoaromatic substrates, using allylamine as a nucleophile, allowed optimising the first step of the sequential process and provided the access to a set of allylcarboxamide products.

In order to minimise product losses by work-up and purification procedures, a sequential catalytic aminocarbonylation/hydroformylation methodology was then carried out, without work-up or purification of the allylcarboxamide intermediates. Thus, using iodobenzene (1) as a model substrate, the crude mixture obtained in the microwave-assisted aminocarbonylation step was directly subjected to hydroformylation conditions ( 8 bar $\mathrm{H}_{2} / \mathrm{CO}$ ) with a rhodium/triphenylphosphine catalyst, at $50{ }^{\circ} \mathrm{C}$. However, in a first attempt, no conversion was observed after $6 \mathrm{~h}$. This was attributed to the formation of a sterically hindered rhodium/DBU complex of type $\mathbf{1 7}$ [65,66], which leads to formation of catalytically inactive species.

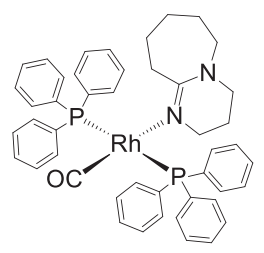

17

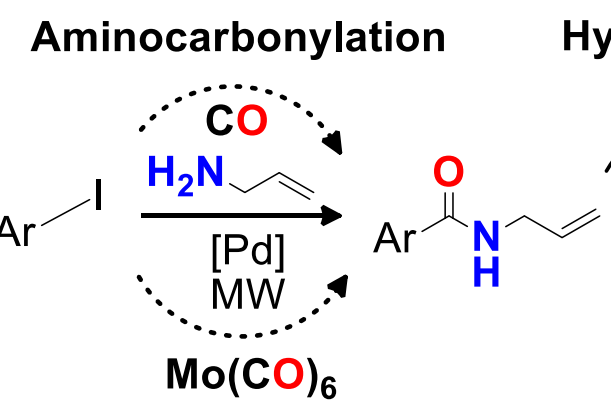

Hydroformylation

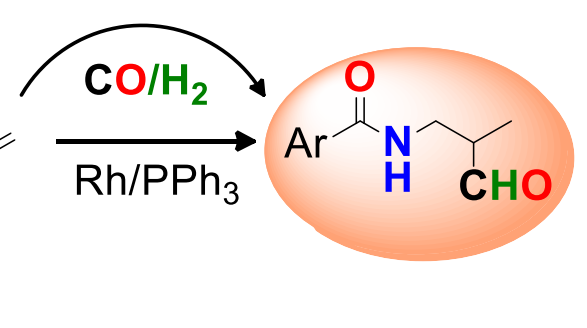

Scheme 1. Sequential aminocarbonylation/hydroformylation approach for the synthesis of formylcarboxamides. 
Table 1

Aminocarbonylation of iodobenzene (1) using allylamine as nucleophile. ${ }^{a}$

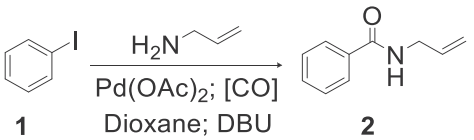

\begin{tabular}{|c|c|c|c|c|c|c|}
\hline Entry & Carbonyl source & Heating & Time (min) & Conversion (\%) & Selectivity (\%) & $\operatorname{TOF}\left(h^{-1}\right)$ \\
\hline 1 & CO (3 bar) & Conv. & 60 & 79 & 69 & 32 \\
\hline 2 & $\mathrm{CO}$ (3 bar) & MW (150 W) & 5 & 76 & 62 & 365 \\
\hline 3 & $\mathrm{Mo}(\mathrm{CO})_{6}$ & Conv. & 60 & 99 & 82 & 40 \\
\hline 4 & $\mathrm{Mo}(\mathrm{CO})_{6}$ & MW (150 W) & 5 & 99 & 76 & 475 \\
\hline
\end{tabular}

${ }^{\mathrm{a}}$ Reaction conditions: iodobenzene $(0.4 \mathrm{mmol})$, allylamine $(0.6 \mathrm{mmol}), \mathrm{Pd}(\mathrm{OAc})_{2}(0.01 \mathrm{mmol}), \mathrm{Mo}(\mathrm{CO}){ }_{6}(0.4 \mathrm{mmol}), \mathrm{DBU}(0.2 \mathrm{~mL}), \mathrm{dioxane}(3 \mathrm{~mL}), 125{ }^{\circ} \mathrm{C}$.

To overcome this issue, the crude mixture, resulting from the aminocarbonylation step, was passed through a silica-pad prior to hydroformylation. Following this procedure, full substrate conversion was obtained in $5 \mathrm{~h}$, with $72 \%$ chemoselectivity for oxoproducts and $44 \%$ regioselectivity for the branched aldehyde (18) (Table 3, entry 1 ). Then, aiming the preparation of biologically relevant formylcarboxamide molecules, the scope of this sequential aminocarbonylation/hydroformylation methodology was expanded to iodopyridine (5), iodopyrazoline (8) and iodochalcone (9) substrates.

In a typical sequential aminocarbonylation/hydroformylation experiment, the iodo-substrate, allylamine, $\mathrm{Pd}(\mathrm{OAc})_{2}, \mathrm{Mo}(\mathrm{CO})_{6}$ and DBU were dissolved in dioxane and placed inside a microwave vial. The mixture was then subjected to microwave irradiation $(\mathrm{P}=150 \mathrm{~W})$, the reaction proceeding at $125{ }^{\circ} \mathrm{C}$ for $5 \mathrm{~min}$. After cooling down to room temperature, the obtained mixture was filtered through a silica pad and the residue was introduced via cannula into an autoclave containing the precursor, $\mathrm{Rh}(\mathrm{CO})_{2} \mathrm{acac}$ and triphenylphosphine $\left(\mathrm{PPh}_{3}\right)$. The autoclave was then pressurised with 8 bar $\mathrm{H}_{2} / \mathrm{CO}$ and kept at $50{ }^{\circ} \mathrm{C}$ for $5 \mathrm{~h}$ (or $12 \mathrm{~h}$ ). At the end, the solvent was evaporated and the crude mixture was analysed by ${ }^{1} \mathrm{H}$ and ${ }^{13} \mathrm{C}$ NMR spectroscopy.

The sequential aminocarbonylation/hydroformylation processes proceeded with complete conversions and the chemoselectivity for oxo-products was in the range $56-73 \%$. The regioselectivity for the branched aldehydes varied from 36 to 51\% (Table 3, entries 2-4). Upon purification by column chromatography in silica gel, the branched formylcarboxamide products 18-21 were isolated in yields ranging from 19 to $22 \%$. These low yields can be explained by the significant formation of linear aldehydes and the respective cyclisation products (not isolated), resultant from intramolecular condensation between the carboxamide and formyl moiety of the linear aldehydes, which leads to the formation of a stable 5 membered ring (Scheme 2) [67], evidenced by ${ }^{1} \mathrm{H}$ NMR and GC-MS.

It is worth mentioning that, in the case of chalcone-derived substrate (9), the hydroformylation of the allyl moiety occurred concomitantly with reduction of the conjugated internal $\mathrm{C}=\mathrm{C}$ double bond, which resulted in the formation of formylcarboxamide 21, obtained as major product (Table 3, entry 4).

\section{Conclusions}

We have developed an innovative catalytic aminocarbonylation/ hydroformylation sequential approach to prepare new formylcarboxamide molecules. The optimisation of the first step of the catalytic sequence was achieved through microwave-assisted palladium-catalysed aminocarbonylation of iodoaromatic substrates, using allylamine as nucleophile and $\mathrm{Mo}(\mathrm{CO})_{6}$ as alternative carbonyl source, which provided an efficient strategy for the synthesis of allylcarboxamide derivatives. The subsequent $\mathrm{Rh} /$ triphenylphosphine-catalysed hydroformylation of the allylcarboxamide intermediates allowed the synthesis of a set of new $\mathrm{N}$ heterocyclic and chalcone-based formylcarboxamides, including 6chloro- $N$-(2-methyl-3-oxopropyl)nicotinamide, 4-(1,3-diphenyl4,5-dihydro- $1 \mathrm{H}$-pyrazol-5-yl)- $\mathrm{N}$-(2-methyl-3-oxopropyl)benzamide and (E)-N-(2-methyl-3-oxopropyl)-4-(3-oxo-3-phenylprop1-en-1-yl)benzamide. In sum, this transition-metal catalysed aminocarbonylation/hydroformylation sequential process opens new perspectives regarding the preparation of biologically relevant molecules containing both carboxamide and formyl motifs.

\section{Experimental section}

\subsection{General}

Manipulation of all moisture sensitive reagents was carried out under nitrogen atmosphere by using Schlenk techniques. Glassware was dried in an oven at $200{ }^{\circ} \mathrm{C}$ and cooled under a nitrogen atmosphere. Palladium (II) acetate, triphenylphosphine, $\mathrm{Mo}(\mathrm{CO})_{6}$, iodobenzeno, 1-bromo-4-iodobenzene, 4-methoxyiodobenzene, 2chloro-5-iodopyridine, 7-iodoindole, 5-iodoindole, DBU and dioxane were purchased from Merck and used without further purification. The iodopyrazoline $\mathbf{8}$ and iodochalcone $\mathbf{9}$ substrates were synthesised through a mechanochemical synthetic methodology, recently developed by some of us [68].

Microwave-assisted experiments were performed in thickwalled glass vials under closed-vessel conditions, using a CEM Discover ${ }^{\circledR}$ SP Focused Microwave ${ }^{\text {TM }}$ Synthesis System. NMR spectra were recorded on a Bruker Avance 400 spectrometer, operating at $400.13 \mathrm{MHz}$ for ${ }^{1} \mathrm{H}$ NMR and $100.62 \mathrm{MHz}$ for ${ }^{13} \mathrm{C}$ NMR. Chemical shifts $(\delta)$ are reported in ppm relatively to $\mathrm{CDCl}_{3}$ (7.260 and $77.16 \mathrm{ppm}$ for ${ }^{1} \mathrm{H}$ and ${ }^{13} \mathrm{C}$, respectively) or tetramethylsilane (TMS). High-resolution mass spectrometry analysis was carried out on a Bruker Microtof apparatus, equipped with selective ESI detector. Samples of the reactions were analysed by: a) gas chromatography carried out on Agilent-7820A GC System equipped with a non-polar capillary HP-5 column (5\% diphenyl and 95\% dimethylpolysiloxane), with $30 \mathrm{~m}$ length and $0.32 \mathrm{~mm}$ inside diameter and using nitrogen as carrier gas, and equipped with an FID detector; or b) gas chromatography coupled with mass spectrometry (GC-MS) using an Agilent 7820A GC System, equipped with a HP-5 MS column, coupled to an Agilent 5975 MSD System Technologies spectrometer, using EI (70 eV) and helium as carrier gas. Carboxamide derivatives were analysed by gas chromatography using the following methods: a) GC-FID method: injector temp $250{ }^{\circ} \mathrm{C}$, oven: starting temp $50{ }^{\circ} \mathrm{C}$ (hold-time $2 \mathrm{~min}$ ), heating rate $30^{\circ} \mathrm{C} \mathrm{min}-1$, final temp $280{ }^{\circ} \mathrm{C}$ (hold-time $9 \mathrm{~min}$ ); detector temp $280{ }^{\circ} \mathrm{C}$, carrier gas: 
Table 2

Microwave-assisted Pd-catalysed aminocarbonylation using allylamine as nucleophile. ${ }^{a}$

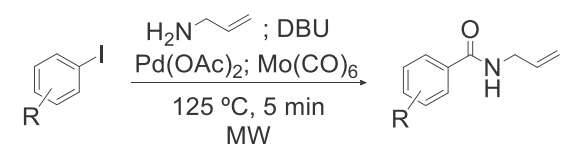

Entry Substrate

3

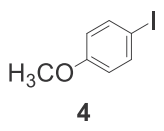

4

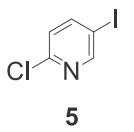

5

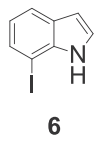

6

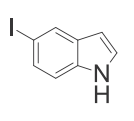

$7^{c}$

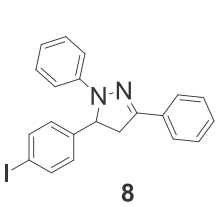

8

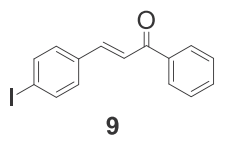

Product (yield, \%)
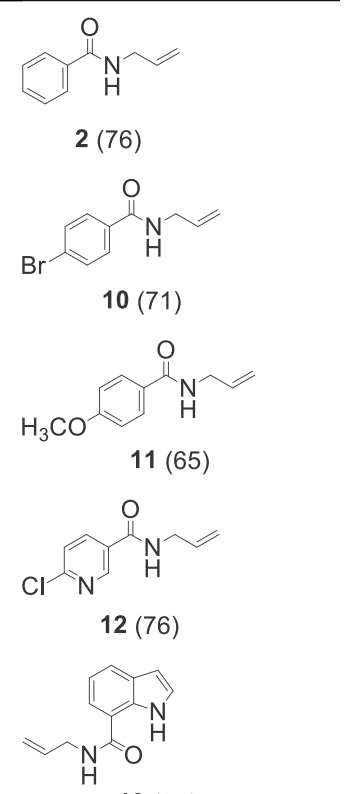

$13(87)$
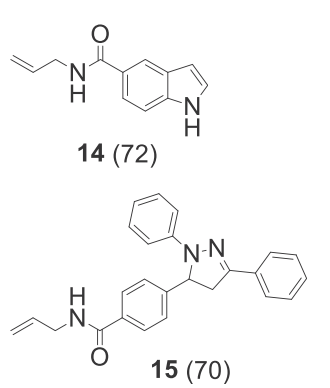

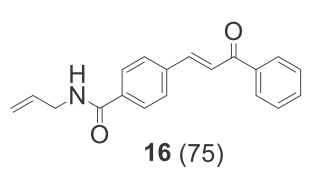

${ }^{a}$ Reaction conditions: substrate $(0.4 \mathrm{mmol})$, allylamine $(0.6 \mathrm{mmol}), \mathrm{Pd}(\mathrm{OAc})_{2}$ (0.01 mmol), $\mathrm{Mo}(\mathrm{CO})_{6}(0.4 \mathrm{mmol}), \mathrm{DBU}(0.2 \mathrm{~mL})$, dioxane $(3 \mathrm{~mL}), 125{ }^{\circ} \mathrm{C}, 5 \mathrm{~min}$, $150 \mathrm{~W}$.

b Yield determined by GC-MS analysis of crude mixtures.

c $1 \mathrm{~h}$.

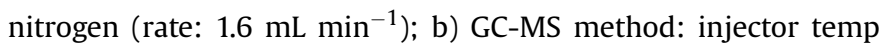
$250^{\circ} \mathrm{C}$, oven: starting temperature $50^{\circ} \mathrm{C}$ (hold-time $1 \mathrm{~min}$ ), heating rate $15{ }^{\circ} \mathrm{C} \mathrm{min}-1$, final temp $280{ }^{\circ} \mathrm{C}$ (hold-time $15 \mathrm{~min}$ ); detector temp $280^{\circ} \mathrm{C}$, carrier gas: helium (rate: $1 \mathrm{~mL} \mathrm{~min}^{-1}$ ).

\subsection{Microwave-assisted palladium-catalysed aminocarbonylation}

The iodoaryl substrate $(0.4 \mathrm{mmol})$, allylamine ( $45 \mu \mathrm{L}, 0.6 \mathrm{mmol})$, $\mathrm{Pd}(\mathrm{OAc})_{2}$ (2.2 mg, $\left.0.01 \mathrm{mmol}\right), \mathrm{Mo}(\mathrm{CO})_{6}(105.6 \mathrm{mg}, 0.4 \mathrm{mmol}), \mathrm{DBU}$ $(180 \mu \mathrm{L}, 1.2 \mathrm{mmol}$ ) were dissolved in dioxane $(3 \mathrm{~mL})$ and placed into a microwave vial. The mixture was subjected to microwave irradiation (Initial Power $=150 \mathrm{~W}$ ) and the reaction was conducted at $125{ }^{\circ} \mathrm{C}$ for $5 \mathrm{~min}$. The mixture was then cooled to room temperature, filtered through a small plug of Celite and evaporated to dryness with the crude being analysed by GC-MS and/or GC-FID (methods described in Section 5.1). The residue obtained was dissolved in dichloromethane $(20 \mathrm{~mL})$ and washed with water $(3 \times 20 \mathrm{~mL})$. The organic phase was dried over anhydrous $\mathrm{Na}_{2} \mathrm{SO}_{4}$, filtered and evaporated to a solid material or to a waxy residue. The reaction products were isolated and purified by column chromatography (Silicagel 60 (Merck), 0.063-0.200 mm), using EtOAc/nhexane or EtOAc/ $\mathrm{CH}_{2} \mathrm{Cl}_{2}$ mixtures as the eluents (specified below for each compound).

\subsection{General procedure of sequential aminocarbonylation/ hydroformylation}

For the sequential aminocarbonylation/hydroformylation process the iodoaromatic substrate $(0.4 \mathrm{mmol})$, allylamine $(45 \mu \mathrm{L}$, $0.6 \mathrm{mmol}, 1.5$ equiv. $), \mathrm{Pd}(\mathrm{OAc})_{2}(2.2 \mathrm{mg}, 0.01 \mathrm{mmol}), \mathrm{Mo}(\mathrm{CO})_{6}$ (105.6 mg, $0.4 \mathrm{mmol}), \mathrm{DBU}(180 \mu \mathrm{L}, 1.2 \mathrm{mmol})$ and dioxane $(3 \mathrm{~mL})$ were placed into a microwave vial. The mixture was subjected to microwave irradiation (Initial Power $=150 \mathrm{~W}$ ) and the reaction was conducted at $125{ }^{\circ} \mathrm{C}$ for $5 \mathrm{~min}$. After cooled down to room temperature, the aminocarbonylation crude mixture was filtered through a small plug of silica gel and introduced via cannula into the autoclave. The rhodium precursor $\mathrm{Rh}(\mathrm{acac})(\mathrm{CO})_{2}$ $\left(4 \times 10^{-3} \mathrm{mmol}, 1.03 \mathrm{mg}\right)$ and triphenylphosphine $(0.02 \mathrm{mmol}$, $5.24 \mathrm{mg}$ ) were dissolved in dioxane $(3 \mathrm{~mL})$, under argon atmosphere, and introduced via cannula into the autoclave. After purging the system with three cycles of vacuum/syngas the reactor was pressurised with 8 bar of $\mathrm{H}_{2} / \mathrm{CO}(1: 1)$ and kept at $50{ }^{\circ} \mathrm{C}$. After $5 \mathrm{~h}$, the reactor was cooled to room temperature and slowly depressurised. The reaction mixture was evaporated to dryness analysed by ${ }^{1} \mathrm{H}$ NMR spectroscopy. The reaction products were isolated and purified by column chromatography (Silicagel 60 (Merck), 0.063-0.200 mm), using EtOAc/n-hexane or EtOAc $/ \mathrm{CH}_{2} \mathrm{Cl}_{2}$ mixtures as the eluents (specified below for each compound).

\subsubsection{Characterisation of products}

The spectroscopic data of $\mathbf{2 , 1 0}$ and $\mathbf{1 1}$ are in agreement with that previously reported $[69,70,71]$.

\subsubsection{N-allyl-6-chloronicotinamide (12). $\mathrm{Rf} \quad\left(\mathrm{CH}_{2} \mathrm{Cl}_{2} / \mathrm{EtOAC}\right.$} $1: 1)=0.74$. Beige solid, Yield 23\%, $18 \mathrm{mg}$ (0.09 mmol). ${ }^{1} \mathrm{H}$ RMN (400 MHz, $\left.\mathrm{CDCl}_{3}, \mathrm{ppm}\right): \delta 8.75(\mathrm{~d}, J=7.6 \mathrm{~Hz}, 1 \mathrm{H}), 8.10$ (dd, $J=8.3$, $2.5 \mathrm{~Hz}, 1 \mathrm{H}), 7.43(\mathrm{~d}, J=8.3 \mathrm{~Hz}, 1 \mathrm{H}), 6.18(\mathrm{~s}, 1 \mathrm{H}), 5.97-5.90(\mathrm{~m}, 1 \mathrm{H})$, $5.28(\mathrm{dd}, J=17.1,1.2 \mathrm{~Hz}, 1 \mathrm{H}), 5.23(\mathrm{dd}, J=10.2,0.9 \mathrm{~Hz}, 1 \mathrm{H})$, 4.12-4.08 (m, 2H). ${ }^{13} \mathrm{C}$ RMN (100 MHz, $\left.\mathrm{CDCl}_{3}, \mathrm{ppm}\right): \delta 164.5 ; 154.5$; $148.0 ; 138.1 ; 133.6 ; 129.2 ; 124.6 ; 117.6 ; 42.8$. HRMS (ESI) $(\mathrm{m} / z)$ : found 197.0477 $[\mathrm{M}+\mathrm{H}]^{+}$(calcd. 197.0476).

4.3.1.2. $N$-allyl-1H-indole-7-carboxamide (13). Rf (EtOAc/n-hex $1: 4)=0.37$. White solid. Yield $20 \%, 16 \mathrm{mg}$ (0.08 mmol). ${ }^{1} \mathrm{H}$ RMN (400 MHz, $\mathrm{CDCl}_{3}, \mathrm{ppm}$ ): $\delta 10.32$ (br s, $\left.1 \mathrm{H}\right), 7.81$ (d, $J=7.8 \mathrm{~Hz}, 1 \mathrm{H}$ ), $7.38(\mathrm{~d}, J=7.4 \mathrm{~Hz}, 1 \mathrm{H}), 7.33-7.32(\mathrm{~m}, 1 \mathrm{H}), 7.12(\mathrm{t}, J=7.7 \mathrm{~Hz}, 1 \mathrm{H})$, 6.58-6.57 (m, 1H), $6.47(\mathrm{~s}, 1 \mathrm{H}), 6.01-5.94(\mathrm{~m}, 1 \mathrm{H}), 5.30$ (dd, $J=17.2$, $1.4 \mathrm{~Hz}, 1 \mathrm{H}), 5.21$ (dd, $J=10.2,1.3 \mathrm{~Hz}, 1 \mathrm{H}), 4.16-4.13(\mathrm{~m}, 2 \mathrm{H}) .{ }^{13} \mathrm{C}$ RMN (100 MHz, $\left.\mathrm{CDCl}_{3}, \mathrm{ppm}\right): \delta 167.8 ; 135.6 ; 134.4 ; 129.7 ; 125.8$; $125.0 ; 118.9 ; 118.8 ; 116.8 ; 115.9 ; 102.1 ; 42.2$. HRMS (ESI) $(\mathrm{m} / \mathrm{z})$ : found $201.1023[\mathrm{M}+\mathrm{H}]^{+}$(calcd. 201.1022).

4.3.1.3. $\mathrm{N}$-allyl-1H-indole-5-carboxamide (14). $\mathrm{Rf} \quad\left(\mathrm{CH}_{2} \mathrm{Cl}_{2} / \mathrm{EtOAC}\right.$ $2: 1)=0.51$. White solid. Yield $23 \%, 18 \mathrm{mg}(0.09 \mathrm{mmol}) .{ }^{1} \mathrm{H}$ RMN (400 MHz, $\left.\mathrm{CDCl}_{3}, \mathrm{ppm}\right): \delta 8.53$ (br s, $\left.1 \mathrm{H}\right), 8.11$ (s, $\left.1 \mathrm{H}\right), 7.66$ (dd, $J=8.5,1.5 \mathrm{~Hz}, 1 \mathrm{H}), 7.42(\mathrm{~d}, J=8.5 \mathrm{~Hz}, 1 \mathrm{H}), 7.28-7.26(\mathrm{~m}, 1 \mathrm{H}), 6.62(\mathrm{~s}$, 
Table 3

Synthesis of formylcarboxamides by sequential aminocarbonylation/hydroformylation methodology.

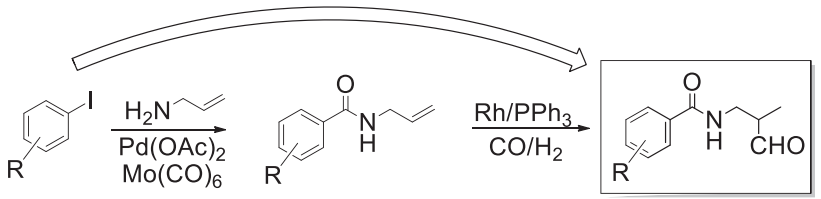

\begin{tabular}{|c|c|c|c|c|}
\hline Entry & Substrate & Chemo (\%) & iso-Regio (\%) & $\begin{array}{c}\text { Product } \\
\text { (isolated yield, \%) }\end{array}$ \\
\hline 1 & 1 & 72 & 44 & $\begin{array}{l}\mathrm{N} \\
\mathrm{H}\end{array}$ \\
\hline 2 & 5 & 73 & 51 & $19(20)$ \\
\hline $3^{\mathrm{d}}$ & 8 & 56 & 46 & $20(19)$ \\
\hline $\mathbf{4}^{\mathrm{d}}$ & $\mathrm{O}$ & 64 & 36 & $21(22)$ \\
\hline
\end{tabular}

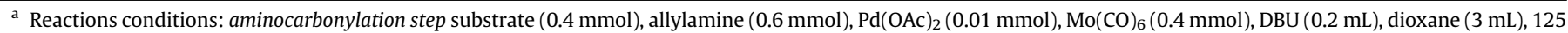
${ }^{\circ} \mathrm{C}, 150 \mathrm{~W}, 5 \mathrm{~min}$,; hydroformylation step: $\mathrm{Rh}(\mathrm{acac})(\mathrm{CO})_{2} / \mathrm{PPh}_{3}=1: 5 ; \mathrm{Rh} /$ substrate $=1: 100 ; \mathrm{P}=8$ bar $\mathrm{H}_{2} / \mathrm{CO} ; 50{ }^{\circ} \mathrm{C}, 5$ h. Conversion was ca $99 \%$ in all cases.

b Chemoselectivity for oxo-products (determined by ${ }^{1} \mathrm{H}$ NMR).

${ }^{c}$ Regioselectivity for the branched aldehyde (iso) (determined by ${ }^{1} \mathrm{H}$ NMR).

d $12 \mathrm{~h}$.<smiles>O=CCCCNC(=O)[AlH]O</smiles>

Scheme 2. Formation of 5-membered ring by intramolecular condensation.

$1 \mathrm{H}), 6.23(\mathrm{br} \mathrm{s}, 1 \mathrm{H}), 6.01-5.94(\mathrm{~m}, 1 \mathrm{H}), 5.29(\mathrm{dd}, J=17.1,1.5 \mathrm{~Hz}, 1 \mathrm{H})$, $5.19(\mathrm{dd}, J=10.2,1.3 \mathrm{~Hz}, 1 \mathrm{H}), 4.14-4.12(\mathrm{~m}, 2 \mathrm{H}) .{ }^{13} \mathrm{C}$ RMN $(100 \mathrm{MHz}$, $\left.\mathrm{CDCl}_{3}, \mathrm{ppm}\right): \delta 168.6 ; 137.7 ; 134.7 ; 127.7 ; 126.6 ; 125.7 ; 121.2 ; 120.3$; 116.6; 111.2; 103.8; 42.6. HRMS (ESI) $(\mathrm{m} / \mathrm{z})$ : found $201.1024[\mathrm{M}+\mathrm{H}]^{+}$ (calcd. 201.1022).

4.3.1.4. N-allyl-4-(1,3-diphenyl-4,5-dihydro-1H-pyrazol-5-yl)benzamide (15). Rf (EtOAc $/ n$-hex $1: 1)=0.66$. Yellow solid. Yield $21 \%$, $32 \mathrm{mg}(0.08 \mathrm{mmol}) .{ }^{1} \mathrm{H}$ RMN (400 MHz, $\left.\mathrm{CDCl}_{3}, \mathrm{ppm}\right): \delta 7.73(\mathrm{t}$, $J=7.3 \mathrm{~Hz}, 4 \mathrm{H}), 7.31-7.25(\mathrm{~m}, 4 \mathrm{H}), 7.34(\mathrm{~d}, J=7.2 \mathrm{~Hz}, 2 \mathrm{H}), 7.20-7.16$ $(\mathrm{m}, 3 \mathrm{H}), 7.04(\mathrm{~d}, J=7.8 \mathrm{~Hz}, 2 \mathrm{H}), 6.80(\mathrm{t}, J=7.3 \mathrm{~Hz}, 1 \mathrm{H}), 6.19(\mathrm{br} \mathrm{s}, 1 \mathrm{H})$, $5.95-5.88(\mathrm{~m}, 1 \mathrm{H}), 5.30(\mathrm{dd}, J=12.4,7.2 \mathrm{~Hz}, 1 \mathrm{H}), 5.24(\mathrm{dd}, J=17.2$, $1.4 \mathrm{~Hz}, 1 \mathrm{H}), 5.17(\mathrm{dd}, J=10.2,1.2 \mathrm{~Hz}, 1 \mathrm{H}), 4.07-4.04(\mathrm{~m}, 2 \mathrm{H}), 3.86(\mathrm{dd}$, $J=17.0,12.4 \mathrm{~Hz}, 1 \mathrm{H}), 3.11(\mathrm{dd}, J=17.1,7.2 \mathrm{~Hz}, 1 \mathrm{H}) .{ }^{13} \mathrm{C} \mathrm{RMN}(100 \mathrm{MHz}$,
$\left.\mathrm{CDCl}_{3}, \mathrm{ppm}\right): \delta 167.0 ; 146.9 ; 146.3 ; 144.7 ; 134.2 ; 134.0 ; 132.6 ; 129.1$; $128.9 ; 128.7 ; 128.0 ; 126.3 ; 125.9 ; 119.5 ; 116.9 ; 113.5 ; 64.3 ; 43.5$; 42.5. HRMS (ESI) $(\mathrm{m} / \mathrm{z})$ : found $381.1835\left[\mathrm{M}^{+}\right]$(calcd. 381.1841).

4.3.1.5. (E)-N-allyl-4-(3-oxo-3-phenylprop-1-en-1-yl)benzamide (16). Rf (EtOAc/n-hex 1:1) $=0.56$. Yellow solid. Yield 25\%, $29 \mathrm{mg}$ (0.1 mmol). ${ }^{1} \mathrm{H}$ RMN (400 MHz, $\left.\mathrm{CDCl}_{3}, \mathrm{ppm}\right): \delta 8.04-8.02(\mathrm{~m}, 2 \mathrm{H})$, 7.85-7.79 (m, 3H), 7.70 (d, $J=8.2 \mathrm{~Hz}, 2 \mathrm{H}), 7.63-7.59(\mathrm{~m}, 2 \mathrm{H}), 7.55$ (d, $J=10.8 \mathrm{~Hz}, 1 \mathrm{H}), 7.51(\mathrm{~d}, J=7.3 \mathrm{~Hz}, 1 \mathrm{H}), 6.26(\mathrm{~s}, 1 \mathrm{H}), 5.99-5.92$ $(\mathrm{m}, 1 \mathrm{H}), 5.28$ (dd, $J=17.1,1.4 \mathrm{~Hz}, 1 \mathrm{H}), 5.21(\mathrm{dd}, J=10.2,1.2 \mathrm{~Hz}, 1 \mathrm{H})$, 4.13-4.10 (m, 2H). ${ }^{13} \mathrm{C}$ RMN (100 MHz, $\mathrm{CDCl}_{3}$, ppm): $\delta$ 190.4; 166.6; $143.4 ; 138.0 ; 137.9 ; 136.0 ; 134.1 ; 133.2 ; 128.8 ; 128.7 ; 128.6 ; 127.7$; 123.8; 117.0; 42.7. HRMS (ESI) $(\mathrm{m} / \mathrm{z})$ : found $292.13302[\mathrm{M}+\mathrm{H}]^{+}$ (calcd. 292.1332). 
4.3.1.6. $\mathrm{N}$-(2-methyl-3-oxopropyl)benzamide (18). $\mathrm{Rf}\left(\mathrm{CH}_{2} \mathrm{Cl}_{2} / \mathrm{EtOAc}\right.$ $7: 3)=0.54$. Yellow oil. Yield $21 \%, 16 \mathrm{mg}(0.08 \mathrm{mmol}) .{ }^{1} \mathrm{H}$ RMN (400 MHz, $\left.\mathrm{CDCl}_{3}, \mathrm{ppm}\right): \delta 9.71(\mathrm{~s}, 1 \mathrm{H}), 7.74-7.72(\mathrm{~m}, 2 \mathrm{H}), 7.50-7.46$ $(\mathrm{m}, 1 \mathrm{H}), 7.40(\mathrm{dd}, J=10.2,4.6 \mathrm{~Hz}, 2 \mathrm{H}), 6.73(\mathrm{br} \mathrm{s}, 1 \mathrm{H}), 3.75-3.69(\mathrm{~m}$, $1 \mathrm{H}), 3.57-3.50(\mathrm{~m}, 1 \mathrm{H}), 2.82-2.74(\mathrm{~m}, 1 \mathrm{H}), 1.21(\mathrm{~d}, J=7.5 \mathrm{~Hz}, 3 \mathrm{H})$. ${ }^{13} \mathrm{C}$ RMN (100 MHz, $\left.\mathrm{CDCl}_{3}, \mathrm{ppm}\right): \delta$ 204.5; 167.7; 134.3; 131.7; $128.7 ; 127.0 ; 46.9 ; 39.9 ; 11.6$. HRMS (ESI) $(\mathrm{m} / \mathrm{z})$ : found 214.08399 $[\mathrm{M}+\mathrm{Na}]^{+}$(calcd. 214.0839).

\subsubsection{6-chloro-N-(2-methyl-3-oxopropyl)nicotinamide}

(19). Rf $\left(\mathrm{CH}_{2} \mathrm{Cl}_{2} /\right.$ EtOAc $\left.1: 2\right)=0.27$. Beige solid. Yield $20 \%, 18 \mathrm{mg}$ (0.08 mmol). $\left.{ }^{1} \mathrm{H} \mathrm{RMN} \mathrm{(400} \mathrm{MHz,} \mathrm{CDCl}_{3}, \mathrm{ppm}\right): \delta 9.70(\mathrm{~s}, 1 \mathrm{H}), 8.73$ (d, $J=2.2 \mathrm{~Hz}, 1 \mathrm{H}), 8.04$ (dd, $J=8.3,2.5 \mathrm{~Hz}, 1 \mathrm{H}), 7.39$ (d, $J=8.3 \mathrm{~Hz}, 1 \mathrm{H})$, $6.92(\mathrm{~s}, 1 \mathrm{H}), 3.78-3.70(\mathrm{~m}, 1 \mathrm{H}), 3.56-3.48(\mathrm{~m}, 1 \mathrm{H}), 2.86-2.78(\mathrm{~m}$, $1 \mathrm{H}), 1.23$ (d, $J=7.5 \mathrm{~Hz}, 3 \mathrm{H}) .{ }^{13} \mathrm{C}$ RMN (100 MHz, CDCl $\left.3, \mathrm{ppm}\right)$ : $\delta$ 204.6; 164.8; 154.4; 148.2; 139.2; 129.3; 124.8; 46.7; 39.0; 11.7. HRMS (ESI) $(\mathrm{m} / \mathrm{z})$ : found $227.0581[\mathrm{M}+\mathrm{H}]^{+}$(calcd. 227.0582).

4.3.1.8. 4-(1,3-diphenyl-4,5-dihydro-1H-pyrazol-5-yl)-N-(2-methyl3-oxopropyl)benzamide (20). Rf (EtOAc/n-hex 2:1) $=0.44$. Yellow solid. Yield 19\%, $31 \mathrm{mg}(0.08 \mathrm{mmol}) .{ }^{1} \mathrm{H}$ RMN (400 MHz, $\mathrm{CDCl}_{3}$, ppm): $\delta 9.69(\mathrm{~s}, 1 \mathrm{H}), 7.73-7.66(\mathrm{~m}, 4 \mathrm{H}), 7.39-7.34(\mathrm{~m}, 5 \mathrm{H}), 7.19-7.15$ $(\mathrm{m}, 2 \mathrm{H}), 7.03$ (d, $J=7.8 \mathrm{~Hz}, 2 \mathrm{H}), 6.79(\mathrm{t}, J=7.3 \mathrm{~Hz}, 1 \mathrm{H}), 6.60(\mathrm{t}$, $J=5.9 \mathrm{~Hz}, 1 \mathrm{H}), 5.30(\mathrm{dd}, J=12.5,7.0 \mathrm{~Hz}, 1 \mathrm{H}), 3.86(\mathrm{dd}, J=17.1$, $12.4 \mathrm{~Hz}, 1 \mathrm{H}), 3.74-3.67(\mathrm{~m}, 1 \mathrm{H}), 3.53-3.50(\mathrm{~m}, 1 \mathrm{H}), 3.10(\mathrm{dd}, J=17.1$, $7.3 \mathrm{~Hz}, 1 \mathrm{H}), 2.80-2.74(\mathrm{~m}, 1 \mathrm{H}), 1.21$ (d, $J=7.5 \mathrm{~Hz}, 3 \mathrm{H}) .{ }^{13} \mathrm{C}$ RMN (100 MHz, $\left.\mathrm{CDCl}_{3}, \mathrm{ppm}\right): \delta 204.5 ; 167.3 ; 152.3 ; 146.9 ; 146.4 ; 133.8$; $133.8 ; 129.1 ; 128.9 ; 128.7 ; 128.0 ; 126.4 ; 125.9 ; 119.5 ; 113.5 ; 64.3$; 46.9; 43.5; 39.9; 11.6. HRMS (ESI) $(m / z)$ : found $412.2020[\mathrm{M}+\mathrm{H}]^{+}$ (calcd. 412.2020).

4.3.1.9. (E)-N-(2-methyl-3-oxopropyl)-4-(3-oxo-3-phenylprop-1-en1-yl)benzamide (21). Rf $\left(\mathrm{CH}_{2} \mathrm{Cl}_{2} /\right.$ EtOAc $\left.1: 1\right)=0.6$. Yellow solid. Yield 22\%, $28 \mathrm{mg}$ (0.09 mmol). ${ }^{1} \mathrm{H}$ RMN (400 $\mathrm{MHz}, \mathrm{CDCl}_{3}, \mathrm{ppm}$ ): $\delta 9.71(\mathrm{~s}, 1 \mathrm{H}), 7.95-7.93(\mathrm{~m}, 2 \mathrm{H}), 7.67(\mathrm{~d}, J=8.2 \mathrm{~Hz}, 2 \mathrm{H}), 7.56(\mathrm{t}$, $J=7.4 \mathrm{~Hz}, 1 \mathrm{H}), 7.45(\mathrm{t}, J=7.6 \mathrm{~Hz}, 2 \mathrm{H}), 7.30(\mathrm{~d}, J=8.1 \mathrm{~Hz}, 2 \mathrm{H}), 6.63(\mathrm{~s}$, $1 \mathrm{H}$ ), 3.73 (ddd, $J=13.7,6.7,4.3 \mathrm{~Hz}, 1 \mathrm{H}$ ), 3.53 (ddd, $J=13.8,8.0$, $5.8 \mathrm{~Hz}, 1 \mathrm{H}), 3.30(\mathrm{t}, J=7.5 \mathrm{~Hz}, 2 \mathrm{H}), 3.11(\mathrm{t}, J=7.5 \mathrm{~Hz}, 2 \mathrm{H}), 2.81-2.76$ $(\mathrm{m}, 1 \mathrm{H}), 1.22$ (d, $J=7.5 \mathrm{~Hz}, 3 \mathrm{H}) .{ }^{13} \mathrm{C}$ RMN (100 MHz, $\left.\mathrm{CDCl}_{3}, \mathrm{ppm}\right)$ : $\delta$ 204.5; 198.9; $167.5 ; 145.5 ; 136.8 ; 133.3 ; 132.2 ; 128.8 ; 128.8$; $128.1 ; 127.3 ; 46.9 ; 40.0 ; 39.9 ; 30.0 ; 11.6$. HRMS (ESI) $(\mathrm{m} / \mathrm{z})$ : found: $324.1591[\mathrm{M}+\mathrm{H}]^{+}$(calcd. 324.1594).

\section{Declaration of competing interest}

The authors declare that they have no known competing financial interests or personal relationships that could have appeared to influence the work reported in this paper.

\section{Acknowledgements}

This work was supported by national funds from FCT Fundação para a Ciência e a Tecnologia, I.P., through projects UID/ QUI/00313/2019 to the Coimbra Chemistry Centre (CQC), PTDC/ QUI-OUT/27996/2017 (DUALPI) and POCI-01-0145-FEDER-016387 (SunStorage). LD thanks MATIS - Materiais e Tecnologias Industriais Sustentáveis (CENTRO-010145-FEDER- 00014) and FMSR thanks FCT PhD CATSUS program (PD/BD/114340/2016) for funding.

\section{References}

[1] T.N. Reddy, A. Beatriz, V.J. Rao, D.P. de Lima, Carbonyl Compounds'Journey to amide bond formation, Chem. Asian J. 14 (2019) 344-388, https://doi.org/ 10.1002/asia.201801560.

[2] C. De Risi, G.P. Pollini, V. Zanirato, Recent developments in general methodologies for the synthesis of $\alpha$-Ketoamides, Chem. Rev. 116 (2016) 3241-3305, https://doi.org/10.1021/acs.chemrev.5b00443.

[3] L. Kollár (Ed.), Modern Carbonylation Methods, Wiley-VCH, Weinheim, 2008.

[4] S. Zhao, N.P. Mankad, Metal-catalysed radical carbonylation reactions, Catal. Sci. Technol. 9 (2019) 3603-3613, https://doi.org/10.1039/C9CY00938H.

[5] S.T. Gadge, B.M. Bhanage, Recent developments in palladium catalysed carbonylation reactions, RSC Adv. 4 (2014) 10367-10389, https://doi.org/ 10.1039/c3ra46273k.

[6] R. Franke, D. Selent, A. Börner, Applied hydroformylation, Chem. Rev. 112 (2012) 5675-5732, https://doi.org/10.1021/cr3001803.

[7] S. Perrone, L. Troisi, A. Salomone, Heterocycle synthesis through Pd-catalyzed carbonylative coupling, Eur. J. Org Chem. (2019) 4626-4643, https://doi.org/ 10.1002/ejoc.201900439.

[8] X.-F. Wu, H. Neumann, M. Beller, Synthesis of heterocycles via palladiumcatalyzed carbonylations, Chem. Rev. 113 (2013) 1-35, https://doi.org/ $10.1021 / \mathrm{cr} 300100 \mathrm{~s}$.

[9] A. Kumari, R.K. Singh, Medicinal chemistry of indole derivatives: current to future therapeutic prospectives, Bioorg. Chem. 89 (2019) 103021, https:// doi.org/10.1016/j.bioorg.2019.103021.

[10] P. Das, M.D. Delost, M.H. Qureshi, D.T. Smith, J.T. Njardarson, A survey of the structures of US FDA approved combination drugs, J. Med. Chem. 62 (2019) 4265-4311, https://doi.org/10.1021/acs.jmedchem.8b01610.

[11] P.R. Kamath, D. Sunil, A.A. Ajees, K.S.R. Pai, S. Das, Some new indole-coumarin hybrids; Synthesis, anticancer and Bcl-2 docking studies, Bioorg. Chem. 63 (2015) 101-109, https://doi.org/10.1016/j.bioorg.2015.10.001.

[12] F. Nawaz, O. Alam, A. Perwez, M.A. Rizvi, M.J. Naim, N. Siddiqui, F.H. Pottoo, M. Jha, 3'-(4-(Benzyloxy)phenyl)-1'-phenyl-5-(heteroaryl/aryl)-3,4-dihydro$1^{\prime} \mathrm{H}, 2 \mathrm{H}$-[3,4'-bipyrazole]-2-carboxamides as EGFR kinase inhibitors: synthesis, anticancer evaluation, and molecular docking studies, Arch. Pharm. Chem. Life Sci. 353 (2020) 1900262, https://doi.org/10.1002/ardp.201900262.

[13] Z. Zhang, Y.-L. Gu, Z.-Y. Wang, H.-N. Wang, Y. Zhao, X.-M. Chu, C.-Y. Zhang, M.C. Yan, Synthesis and biological evaluation of novel indoleamide derivatives as antioxidative and antitumor agents, J. Heterocycl. Chem. 57 (2020) 1165-1172, https://doi.org/10.1002/jhet.3853.

[14] R. Tamatam, S.S. Panga, Z. Grigory V, S. Gundala, B.R. Nemallapudi, K.P. Vasikarla, P. Adivireddy, P. Venkatapuram, Synthesis, antimicrobial and antioxidant activities of pyrimidinyl benzothiazine carboxamides, Chemistry 4 (2019) 6813-6820, https://doi.org/10.1002/slct.201900525.

[15] C. Thirumurugan, P. Vadivel, A. Lalitha, S. Lakshmanan, Synthesis, characterization of novel quinoline-2-carboxamide based chalcone derivatives and their molecular docking, photochemical studies, Synth. Commun. 50 (2020) 831-839, https://doi.org/10.1080/00397911.2020.1720737.

[16] S. Nisa, M. Yusuf, Synthetic and antimicrobial studies of N-substituted pyrazoline-based new bis heterocycles, J. Heterocycl. Chem. 57 (2020) 2024-2036, https://doi.org/10.1002/jhet.3910.

[17] M.S. Abdelbaset, M. Abdel-Aziz, M. Ramadan, M.H. Abdelrahman, S.N.A. Bukhari, T.F.S. Ali, G.E.-D.A. Abuo-Rahma, Discovery of novel thienoquinoline-2-carboxamide chalcone derivatives as antiproliferative EGFR tyrosine kinase inhibitors, Bioorg. Med. Chem. 27 (2019) 1076-1086, https:// doi.org/10.1016/j.bmc.2019.02.012.

[18] A.C. Brezny, C.R. Landis, Recent developments in the scope, practicality, and mechanistic understanding of enantioselective hydroformylation, Acc. Chem. Res. 51 (2018) 2344-2354, https://doi.org/10.1021/acs.accounts.8b00335.

[19] S.S. Nurttila, P.R. Linnebank, T. Krachko, J.N.H. Reek, Supramolecular approaches to control activity and selectivity in hydroformylation catalysis, ACS Catal. 8 (2018) 3469-4388, https://doi.org/10.1021/acscatal.8b00288.

[20] A. Cunillera, C. Blanco, A. Gual, J.M. Marinkovic, E.J. Garcia-Suarez, A. Riisager, C. Claver, A. Ruiz, C. Godard, Highly efficient Rh-catalysts immobilised by $\pi-\pi$ stacking for the asymmetric hydroformylation of norbornene under continuous flow conditions, ChemCatChem 11 (2019) 2195-2205, https://doi.org/ 10.1002 /cctc. 201900211.

[21] A. Gual, C. Godard, S. Castillón, C. Claver, Highlights of the Rh-catalysed asymmetric hydroformylation of alkenes using phosphorus donor ligands, Tetrahedron Asymmetry 21 (2010) 1135-1146, https://doi.org/10.1016/ j.tetasy.2010.05.037.

[22] F.G. Delolo, E.N. dos Santos, E.V. Gusevskaya, Anisole: a further step to sustainable hydroformylation, Green Chem. 21 (2019) 1091-1098, https:// doi.org/10.1039/c8gc03750g.

[23] A.C. Faria, M.P. de Oliveira, A.C. Monteiro, R.L.V. Mota, K.C.B. Oliveira, E.N. dos Santos, E.V. Gusevskaya, Hydroformylation of recalcitrating biorenewable compounds containing trisubstituted double bonds, Appl. Catal. Gen. 591 (2020) 117406, https://doi.org/10.1016/j.apcata.2019.117406.

[24] P. Pongrácz, B. Bartal, L. Kollár, L.T. Mika, Rhodium-catalyzed hydroformylation in $\gamma$-valerolactone as a biomass derived solvent, J. Organomet. Chem. $847 \quad$ (2017) 140-145, https://doi.org/10.1016/ j.jorganchem.2017.04.029.

[25] P. Pongrácz, H. Szentjóbi, T. Tóth, P. Huszthy, L. Kollár, Enantioselective hydroformylation of styrene in the presence of platinum(II)-monophosphacrown ether complexes, Mol. Catal. 439 (2017) 128-133, https://doi.org/ 10.1016/j.mcat.2017.06.037.

[26] V.M. Fernández-Alvarez, V. de la Fuente, C. Godard, S. Castillón, C. Claver F. Maseras, J.J. Carbó, Pd-catalysed mono- and dicarbonylation of aryl iodides: insights into the mechanism and the selectivity, Chem. Eur J. 20 (2014) 10982-10989, https://doi.org/10.1002/chem.201403022.

[27] M. Gergely, A. Bényei, L. Kollár, 2-Aminobenzimidazole and -benzoxazole as $\mathrm{N}$-nucleophile in palladium-catalysed aminocarbonylation, Tetrahedron 76 
(2020) 131079, https://doi.org/10.1016/j.tet.2020.1310.

[28] P. Szuroczki, L. Molnár, A. Dörnyei, L. Kollár, Facile, high-yielding synthesis of 4-functionalised 1,2,3-triazoles via amino- and aryloxycarbonylation, Chemistry 5 (2020) 448-451, https://doi.org/10.1002/slct.201903801.

[29] L. Kollár, M.G. Varga, A. Dörnyei, A. Takács, Functionalisation of the uracil ring via palladium-catalysed aminocarbonylation, Tetrahedron 75 (2019) 4632-4639, https://doi.org/10.1016/j.tet.2019.07.008.

[30] A. Brennführer, H. Neumann, M. Beller, Palladium-Catalyzed carbonylation reactions of aryl halides and related compounds, Angew. Chem. Int. Ed. 48 (2009) 4114-4133, https://doi.org/10.1002/anie.200900013.

[31] A. Takács, D. Marosvölgyi-Haskó, Z. Kabak-Solt, L. Damas, F.M.S. Rodrigues, R.M.B. Carrilho, M. Pineiro, M.M. Pereira, L. Kollár, Functionalization of indole at $\mathrm{C}-5$ or $\mathrm{C}-7$ via palladium-catalysed double carbonylation. A facile synthesis of indole ketocarboxamides and carboxamide dimers, Tetrahedron 72 (2016) 247-256, https://doi.org/10.1016/j.tet.2015.11.007.

[32] R.M.B. Carrilho, M.M. Pereira, M.J.S.M. Moreno, A. Takács, L. Kollár, A new facile synthesis of steroid dimers containing 17,17'-dicarboxamide spacers, Tetrahedron Lett. 54 (2013) 2763-2765, https://doi.org/10.1016/ j.tetlet.2013.02.108.

[33] R.M.B. Carrilho, A.R. Almeida, M. Kiss, L. Kollár, R. Skoda-Földes, J.M. Dabrowski, M.J.S.M. Moreno, M.M. Pereira, One-step synthesis of dicarboxamides through Pd-catalysed aminocarbonylation with diamines as $\mathrm{N}$ nucleophiles, Eur. J. Org Chem. (2015) 1840-1847, https://doi.org/10.1002/ ejoc.201403444.

[34] R.M.B. Carrilho, A.R. Almeida, M. Kiss, L. Kollár, R. Skoda-Földes, J.M. Dabrowski, M.J.S.M. Moreno, M.M. Pereira, One-step synthesis of dicarboxamides through Pd-catalysed aminocarbonylation with diamines as $\mathrm{N}$ nucleophiles, Eur. J. Org Chem. (2015) 1840-1847, https://doi.org/10.1002/ ejoc.201403444.

[35] Web of Science ${ }^{\mathrm{TM}}, 2020$ accessed on May $27^{\text {th }} 2020$, https://apps. webofknowledge.com/.

[36] J. Biemolt, E. Ruijter, Advances in palladium-catalyzed cascade cyclizations Adv. Synth. Catal. 360 (2018) 3821-3871, https://doi.org/10.1002/ adsc.201800526.

[37] L. Åkerbladh, S.Y. Chow, L.R. Odell, M. Larhed, Synthesis of $4 H$-Benzo[e][1,3] oxazin-4-ones by a Carbonylation-Cyclization Domino Reaction of orthoHalophenols and Cyanamide, ChemistryOpen 6 (2017) 620-628, https:// doi.org/10.1002/open.201700130.

[38] P. Kalck, M. Urrutigoïty, Tandem hydroaminomethylation reaction to synthesize amines from alkenes, Chem. Rev. 118 (2018) 3833-3861, https:// doi.org/10.1021/acs.chemrev.7b00667.

[39] B.P. Bondžić, Rh catalyzed multicomponent tandem and one-pot reactions under hydroformylation conditions, J. Mol. Catal. A: Chem. 408 (2015) 310-334, https://doi.org/10.1016/j.molcata.2015.07.026.

[40] M. Vilches-Herrera, L. Domke, A. Börner, Isomerization-Hydroformylation tandem reactions, ACS Catal. 4 (2014) 1706-1724, https://doi.org/10.1021/ cs500273d.

[41] A. Cunillera, M.D. de los Bernardos, M. Urrutigoïty, C. Claver, A. Ruiz, C. Godard, Efficient synthesis of chiral $\gamma$-aminobutyric esters via direct rhodium-catalysed enantioselective hydroaminomethylation of acrylates, Catal. Sci. Technol. 10 (2020) 630-634, https://doi.org/10.1039/C9CY01797F.

[42] D.E. Fogg, E.N. dos Santos, Tandem catalysis: a taxonomy and illustrative review, Coord. Chem. Rev. 248 (2004) 2365-2379, https://doi.org/10.1016/ j.ccr.2004.05.012.

[43] A.O. Dias, M.G.P. Gutiérrez, J.A.A. Villarreal, R.L.L. Carmo, K.C.B. Oliveira, A.G. Santos, E.N. dos Santos, E.V. Gusevskaya, Sustainable route to biomassbased amines: rhodium catalyzed hydroaminomethylation in green solvents, Appl. Catal. Gen. 574 (2019) 97-104, https://doi.org/10.1016 j.apcata.2019.02.003.

[44] L. Kollár, P. Pongrácz, Tandem hydroformylation/aldol condensation reactions: synthesis of unsaturated ketones from olefins, J. Organomet. Chem. 866 (2018) 184-188, https://doi.org/10.1016/j.jorganchem.2018.04.031.

[45] B. Bartal, G. Mikle, L. Kollár, P. Pongrácz, Palladium catalyzed carbonylations of alkenyl halides with formic acid to get corresponding $\alpha, \beta$-unsaturated carboxylic acids and esters, Mol. Catal. 467 (2019) 143-149, https://doi.org/ 10.1016/j.mcat.2018.12.026.

[46] J. Cao, Z.-J. Zheng, Z. Xu, L.-W. Xu, Transition-metal-catalyzed transfer carbonylation with $\mathrm{HCOOH}$ or $\mathrm{HCHO}$ as non-gaseous $\mathrm{C} 1$ source, Coord. Chem. Rev. 336 (2017) 43-53, https://doi.org/10.1016/j.ccr.2017.01.005.

[47] S.D. Friis, A.T. Lindhardt, T. Skrydstrup, The development and application of two-chamber reactors and carbon monoxide precursors for safe carbonylation reactions, Acc. Chem. Res. 49 (2016) 594-605, https://doi.org/10.1021/ acs.accounts.5b00471.

[48] F. Xu, D. Lib, W. Han, Transition-metal-free carbonylation of aryl halides with arylboronic acids by utilizing stoichiometric $\mathrm{CHCl}_{3}$ as the carbon monoxideprecursor, Green Chem. 21 (2019) 2911-2915, https://doi.org/10.1039/ c9gc00598f.

[49] J.-B. Penga, X. Qia, X.-F. Wu, Recent achievements in carbonylation reactions: a personal account, Synlett 27 (2016), https://doi.org/10.1055/s-00361588351.

[50] R.I. Khusnutdinov, T.M. Oshnyakova, U.M. Dzhemilev, Molybdenum compounds in organic synthesis, Russ. Chem. Rev. 86 (2017) 128-163, https://doi.org/10.1070/RCR4617.

[51] M. Mamone, J. Aziz, J.L. Bescont, S. Piguel, Aminocarbonylation of N-containing heterocycles with aromatic amines using $\mathrm{Mo}(\mathrm{CO}) 6$, Synthesis 50 (2018) 1521-1526, https://doi.org/10.1055/s-0037-1609152.

[52] L. Wu, Q. Liu, R. Jackstell, M. Beller, Carbonylations of alkenes with CO surrogates, Angew. Chem. Int. Ed. 53 (2014) 6310-6320, https://doi.org/10.1002/ anie.201400793.

[53] L. Åkerbladh, L.R. Odell, M. Larhed, Palladium-Catalyzed molybdenum hexacarbonyl-mediated gas-free carbonylative reactions, Synlett 30 (2019) 141-155, https://doi.org/10.1055/s-0037-1610294.

[54] M. Pineiro, L.D. Dias, L. Damas, G.L.B. Aquino, M.J.F. Calvete, M.M. Pereira, Microwave irradiation as a sustainable tool for catalytic carbonylation reactions, Inorg. Chim. Acta. 455 (2017) 364-377, https://doi.org/10.1016/ j.ica.2016.06.043.

[55] C. Oliver Kappe, My twenty years in microwave chemistry: from kitchen ovens to microwaves that aren't microwaves, Chem. Rec. 19 (2019) 15-39, https://doi.org/10.1002/tcr.201800045.

[56] S. Horikoshi, N. Serpone (Eds.), Microwaves in Catalysis Methodology and Applications, Wiley-VCH Verlag GmbH \& Co. KGaA, Weinheim, 2016.

[57] Cini Elena, E. Petricci, M. Taddei, Pd/C catalysis under microwave dielectric heating, Catalysts 7 (2017) 89, https://doi.org/10.3390/catal7030089.

[58] I.M.Z. Fares, A.E.M. Mekky, A.H.M. Elwahy, I.A. Abdelhamid, Microwaveassisted three component synthesis of novel bis-fused quinazolin-8(4H)-ones linked to aliphatic or aromatic spacer via amide linkages, Synth. Commun. 50 (2020) 893-903, https://doi.org/10.1080/00397911.2020.1725575.

[59] A.R. Almeida, R.D. Dias, C.J.P. Monteiro, A.R. Abreu, P.M.P. Gois, J.C. Bayon, M.M. Pereira, Rhodium-catalysed tandem hydroformylation/arylation reaction with Boronic acids, Adv. Synth. Catal. 356 (2014) 1223-1227, https:// doi.org/10.1002/adsc.201300968.

[60] L. Damas, G.N. Costa, J.C. Ruas, R.M.B. Carrilho, A.R. Abreu, G. Aquino, M.J.F. Calvete, M. Pineiro, M.M. Pereira, Microwave assisted reactions of natural oils: transesterification and hydroformylation/isomerization as tools for high-value compounds, Curr. Microw. Chem. 2 (2015) 53-60, https://doi.org/ $10.2174 / 221333560201150212110805$.

[61] F.M.S. Rodrigues, M.J.F. Calvete, C.J.P. Monteiro, S.A.C. Carabineiro, T.M.R. Maria, J.L. Figueiredo, M.M. Pereira, Hydroaminomethylation reaction as powerful tool for preparation of rhodium/phosphine functionalized nanomaterials. Catalytic evaluation in styrene hydroformylation, Catal. Today (2019), https://doi.org/10.1016/j.cattod.2019.05.045 in press.

[62] A.R. Almeida, R.M.B. Carrilho, A.F. Peixoto, A.R. Abreu, A. Silva, M.M. Pereira, Sequential reactions from catalytic hydroformylation toward the synthesis of amino compounds, Tetrahedron 73 (2017) 2389-2395, https://doi.org/ 10.1016/j.tet.2017.03.023.

[63] F.M.S. Rodrigues, P.K. Kucmierczyk, M. Pineiro, R. Jackstell, R. Franke, M. Beller, M.M. Pereira, Dual Rh-Ru catalysts for reductive hydroformylations of olefins to alcohols, Chem. Sus. Chem. 11 (2018) 2310-2314, https://doi.org/10.1002/ CSSc. 201800488.

[64] L. Damas, R.M.B. Carrilho, S.C.C. Nunes, A.A.C.C. Pais, L. Kollar, M. Pineiro, M.M. Pereira, A novel Pd-catalysed sequential carbonylation/cyclization approach toward bis-N-heterocycles: rationalization by electronic structure calculations, R. Soc. open sci. 5 (2018) 181140, https://doi.org/10.1098/ rsos. 181140 .

[65] J. Schniedermeier, H.J. Haupt, New rhodium(I)- $\pi$-chelate complexes with coordinated amidine bases ( $\mathrm{dbu}, \mathrm{dbn}$ ) and their catalytic properties to polymerize phenylacetylene, J. Organomet. Chem. 506 (1996) 41-47, https:// doi.org/10.1016/0022-328X(95)05689-M.

[66] J.V. Barkley, C.J. Davies, B.T. Heaton, C. Jacob, Rhodium complexes with 1,8diazabicyclo[5.4.0] undec-7-ene (dbu): crystal structure of trans-[Rh(CO)(dbu)(PPh3)2]ClO4, J. Chem. Soc. Dalton Trans. (1995) 2861-2863, https:// doi.org/10.1039/DT9950002861.

[67] A.J. von Wangelin, H. Neumann, D. Gördes, S. Klaus, D. Strübing, M. Beller Multicomponent coupling reactions for organic synthesis: chemoselective reactions with amide-aldehyde mixtures, Chem. Eur J. 9 (2003) 4286-4294, https://doi.org/10.1002/chem.200305048.

[68] C. Gomes, C.S. Vinagreiro, L. Damas, G. Aquino, J. Quaresma, C. Chaves, J. Pimenta, J. Campos, M. Pereira, M. Pineiro, Advanced mechanochemistry device for sustainable synthetic processes, ACS Omega 5 (2020) 10868-10877, https://doi.org/10.1021/acsomega.0c00521.

[69] J.-F. Soulé, H. Miyamura, S. Kobayashi, Powerful amide synthesis from alcohols and amines under aerobic conditions catalyzed by gold or gold/iron, -nickel or -cobalt nanoparticles, J. Am. Chem. Soc. 133 (2011) 18550-18553, https:// doi.org/10.1021/ja2080086.

[70] D.F. Harvey, D.M. Sigano, Synthesis of cyclopropylpyrrolidines via reaction of $\mathrm{N}$-allyl- $\mathrm{N}$-propargylamides with a molybdenum carbene complex. Effect of substituents and reaction conditions, J. Org. Chem. 61 (1996) 2268-2272, https://doi.org/10.1021/jo9519930.

[71] N.G. Moon, A.M. Harned, lodine(III)-promoted synthesis of oxazolines from Nallylamides, Tetrahedron Lett. 54 (2013) 2960-2963, https://doi.org/10.1016/ j.tetlet.2013.03.140. 